\title{
Strategic Purchasing: The Experience in England
}

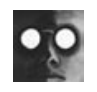

PURCHASING

Gerry McSorley, DBA

Director, Centre for Health Improvement and Leadership, University of Lincoln Visiting Professor in Healthcare Management and Leadership, University of Lincoln

\section{Introduction}

Healthcare policy in the United Kingdom (UK) is placing increasing emphasis on the innovation and creativity of healthcare purchasing for health improvement, as well as on the skills and competencies of those charged with its leadership and management. In considering these developments, the current state of healthcare strategic purchasing in the UK must be framed within a number of key contextual issues:

- The divergent approaches to health policy and its implementation being taken by each of the four different government departments in England, Wales, Northern Ireland and Scotland (Greer 2004)

- The impact of the history of healthcare funding and the current financial climate on the demands placed on those responsible for health purchasing and the relative scope for health improvement that improving or deteriorating levels of funding create (Wanless 2002)

- The central government's broader approach to public services and public services management known as the New Public Management, in which government should "steer" not "row" (Kelman 2005; Pollitt 2002; Osborn and Gaebler 1992)

\section{Health Policy Implementation in the UK}

With the advent of increased devolution of power to the devolved administrations of Scotland, Wales and Northern Ireland, the UK has no single unified approach to health policy or its implementation. I have adapted the following descriptions from Greer (2004): 
- England has opted for markets, hoping that competition between independent trusts (similar to private firms) and between trusts and privately run treatment centres will drive up standards and efficiency. It is hoped that this model will rescue the government from responsibility for every detail of health service delivery.

- Scotland has bet on professionalism, reducing layers of management, placing NHS trusts within integrated boards (along with clinical networks) and increasing the role of professionals in rationing and resource allocation.

- Wales has relied on a professionalist and localist approach, integrating health and local government to improve the coordination of different forms of care at the local level and to raise standards. It is hoped that this will increase local participation in healthcare.

- Northern Ireland has resorted to permissive management, in and out of devolution, concentrating on keeping services going in tough conditions.

The results of such policy divergence among the four UK countries and the relative spend on healthcare might not lead to more activity being delivered, to better population health or to higher levels of public satisfaction. These outcomes might depend more on how resources are deployed and how factors outside the healthcare system influence health (Alvarez-Rosete et al. 2005).

Notwithstanding the nature of this policy divergence, the four countries still share a number of critical challenges:

- Demographic changes - in particular the proportional rise of the elderly population

- Increasing technological developments and associated costs, both human and financial

- Finite resources to fund healthcare

- Potentially unlimited demand for healthcare

- The prevention of unnecessary hospital admission

- Correctly incentivizing the system so as to optimize the chance of achieving the health system's goals

In England, the overall spend on the National Health Service (NHS) has historically been below continental European levels. While many might argue that this comparator is inappropriate given the variations among health systems - some of which are based on social insurance or mixtures of social insurance and private care - the British government committed itself to achieving the target of matching continental European levels of healthcare spending by 2008. This commitment is coupled with the impact of the Treasury forecast of slowing growth in further investment in health services arising from Wanless's Securing Our Future review (2002).

In this paper I place particular emphasis on the situation in England. This is not because that country has the right or wrong solution to strategic purchasing. Rather, given that the majority of the British population lives in England, that is the country in which the greatest impact of health policy reforms will fall.

\section{Background to the Current Situation in England}

Since its election to power in 1997, the Labour government's policy for the English NHS has moved through a number of initiatives and positions. On election, the key attribute was fiscal prudence, largely accepting the previous Conservative government's 
somewhat constraining spending policy. General practice fundholding, a major plank of the Conservative government's reform program, was scrapped. A New Commission for Health Improvement was created to enhance the drive for quality. Many have argued that these early reforms were merely stop-gap measures pending agreement on the future ways forward (Klein 2001).

The first major directional vision was therefore contained in The NHS Plan, which was published with considerable professional support and laid out a 10-year strategy to modernize health services (Department of Health 2000). The key tenets of this plan were as follows:

- "Investment accompanied by reform" (the plan's mission phrase)

- Extra acute hospital beds

- New hospitals funded through the Private Finance Initiative

- Extra staff members who were paid more money

- Creation of the "earned autonomy" concept for hospitals, which provided those hospitals judged to be successful with greater scope to innovate

- Creation of a Modernisation Agency to spread best practices

- Bringing together local municipal services and the NHS to pool resources (e.g., creation of care trusts to commission health and social care in a single organization)

- Extended roles for nurses and allied health professionals

- Creation of patient advocacy and liaison services in each healthcare organization

- A national patient survey

- Reaching a concordat with private sector providers with a view to ending the historical divide between private and public sector providers

- $\quad$ Setting new wait time targets
- New investment and service delivery programs in cancer, coronary heart disease and healthcare for older people

Over time, however, the sense at the government level was that, while The NHS Plan had considerable support, the NHS was not moving quickly enough to modernize and meet patients' and the public's expectations of care and access. The NHS Plan was therefore followed up with the NHS Improvement Plan in 2004 (Department of Health 2004), which added new reform components, including the following:

- Reforming both the supply side and the demand side

- Introducing patient choice of acute sector providers

- Revising the role of the New Commission for Health Improvement to an inspectorate role as the Commission for Healthcare Audit and Inspection

- Setting challenging new targets for access time for diagnosis and treatment

- Advocating the adoption of the triangle of care, with self-management of health by far the most potent proportion of care, followed by disease management (i.e., more proactive support) and, finally, case management for the smallest group of patients with complex needs (i.e., active and specialist care)

The recognition of the divergence between the supply and demand sides embodied by the purchaser-provider split indicated the need for vision and skills on both sides if major progress were to be made in healthcare reform, efficiency and productivity.

The final piece of the reform jigsaw

- Creating a Patient-Led NHS (Department of Health 2005) - saw the culmination of growing concerns about the unbalanced nature 
Figure 1. Organising Framework for NHS Reforms (Healthcare Financial Management Association 2006)

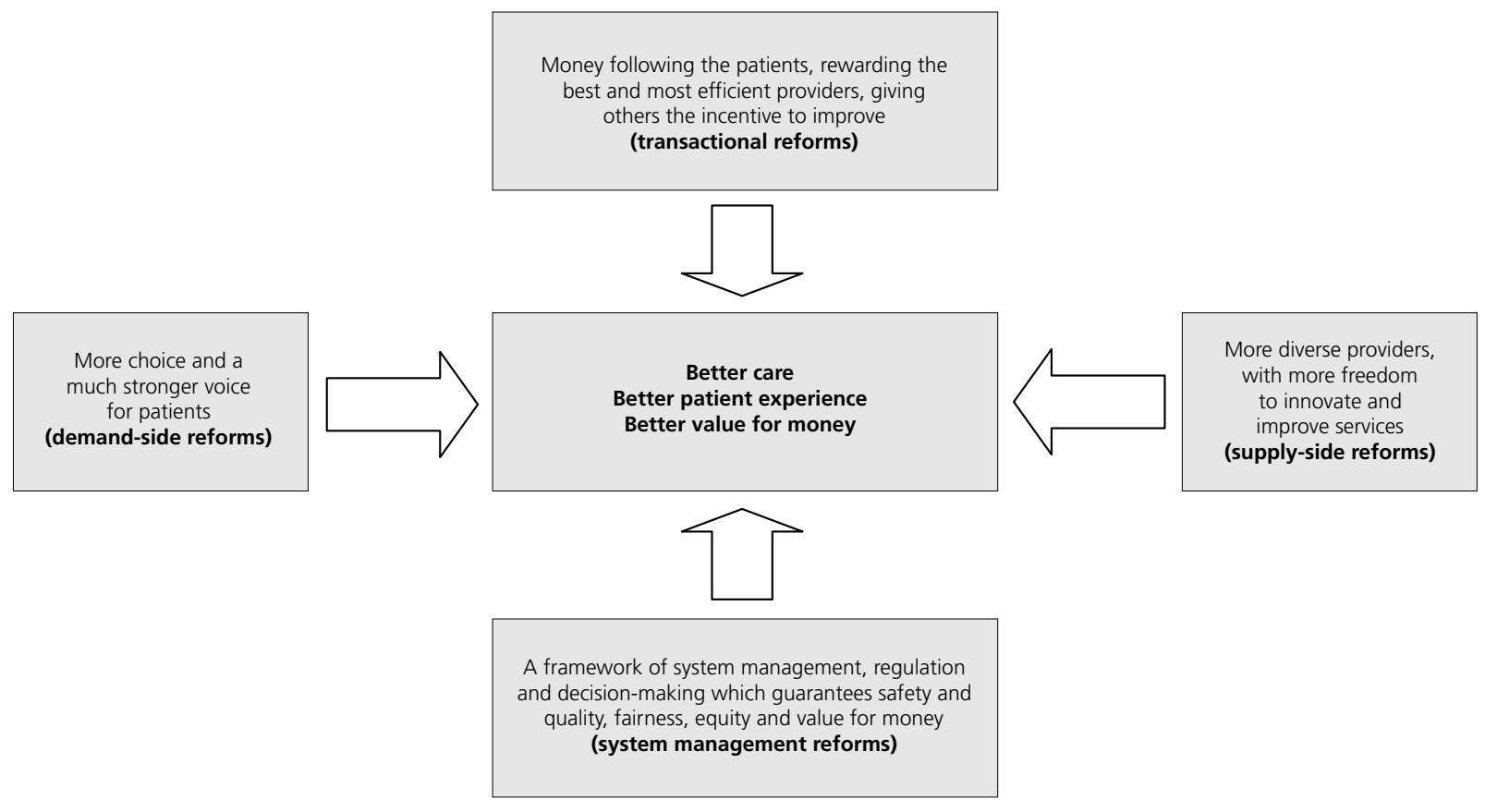

of the power of the NHS supply side in the absence of assertive and skilled purchasing (or "commissioning," as it will be referred to in the rest of this paper). Creating a PatientLed NHS sprang from a recognition that the main focus since 1997 had been the acute care sector, incentivizing it to reduce wait times and to improve its services. In doing so, there was a clear risk that a strong, vibrant, incentivized hospital sector would suck all investment into hospital care unless it was balanced by an equally strong and vibrant commissioning function. Creating a Patient-Led NHS focused specifically on the importance of expert, imaginative commissioning. It promoted the belief that a revitalized commissioning function must represent patients, centre on prevention and public health and ensure hospital providers deliver good value for money.

Figure 1 captures the resulting model, based on the reforms of 2000, 2004 and 2005. The Organising Framework for NHS Reforms was based on the political need to devolve power at the same time as introducing market-style incentives, which were stimulated by allowing patients to choose which providers to visit (whether not-for-profit or for-profit private hospitals, or public hospitals in the shape of foundation hospitals operating in a quasi-commercial fashion). In order to provide that better balance of skills on the commissioning side of the equation, the Department of Health committed itself to devolving $75 \%$ of the NHS budget directly to primary care trusts (PCTs), with a further level of devolution to individual practices in the form of practice-based commissioning $(\mathrm{PBC})$. The unit of currency in this new model was the patient. The method of payment was the tariff: a fixed price for individual procedures based on diagnostic-related groups (DRGs) but adapted to UK clinical practice and renamed healthcare resource groups (HRGs).

This combination of demand- and supplyside reforms has resulted in NHS England 
in 2007 being in a state of transition from a public monopoly insurer and provider of healthcare, governed from Whitehall, to an insurer with devolved commissioning from a mixed market of providers. The question follows, therefore, as to where on a spectrum of market models NHS England will rest? At one end is a wholly market-based health system in which competition rules and regulation is light; at the other end is a system that is nationally planned, owned, provided and governed from the centre. One way to answer the question would be to consider the following criteria (Lewis and Dixon 2005):

- The government's values and ideology

- How the existing reform program is led and managed

- The level of political support for changes

- Public perceptions of the reform program

- Evidence of improvements in patient care

- Public satisfaction with local health services when compared with other developed countries

In essence, the Labour government's philosophy appears to be "what counts is what works" (Greer 2004: 225). Stevens (2004: 42) offers an alternative perspective on such a multidimensional approach to health policy, describing the need to overcome the inertia inherent in all human systems as "constructive discomfort."

Finally, the Organising Framework for NHS Reforms considered the need for system-management reforms. Organizationally, Creating a Patient-Led NHS required changes to the structures underpinning excellent commissioning and to the mechanism by which the Department of Health devolved market management to the regional level. Figures 2 and 3 illustrate the main functions of the new strategic health authorities (SHAs) - overseeing millions of people - and PCTs - delivering services to hundreds of thousands of people (see Department of Health 2006b).

Figure 2. The three main functions of SHAs

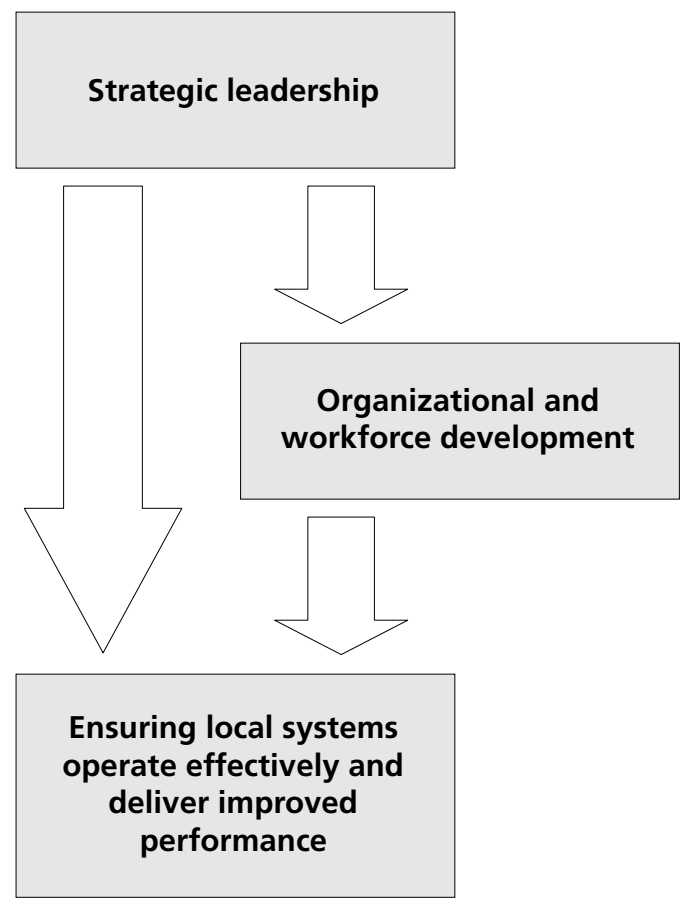

\section{Strategic Commissioning}

Definitional clarity is needed in any debate about the value of commissioning. Such clarity requires answering the following three questions:

- What is commissioning?

- What does it involve?

- What does it seek to achieve?

The Department of Health (2006a:

3) defines commissioning as "the means by which we secure the best value for patients and taxpayers." By "best value," the Department of Health means "the best possible health outcomes, including reduced health inequalities" and "the best possible healthcare" 
delivered "within the resources made available by the taxpayer.” In 1995, Øvretveit provided an alternative definition of commissioning as a sophisticated and strategic process of assessing health needs, developing new services or providers, contracting for services and undertaking a range of strategic efforts to improve population health. Work by Smith and Mays (cited in Wade et al. [2006]) provides a further characterization of commissioning:

- It has a conscience, setting out "how things should be" (i.e., what the system aims to achieve and how).

- It has eyes and ears, observing and reporting on "how things are" (i.e., what the system is currently delivering).

- It has a brain, processing information from both sources (i.e., identifying and implementing the optimal solutions for delivering stated objectives).

\section{Figure 3. The three main functions of PCTs}

Engaging with local populations to improve health and well-being

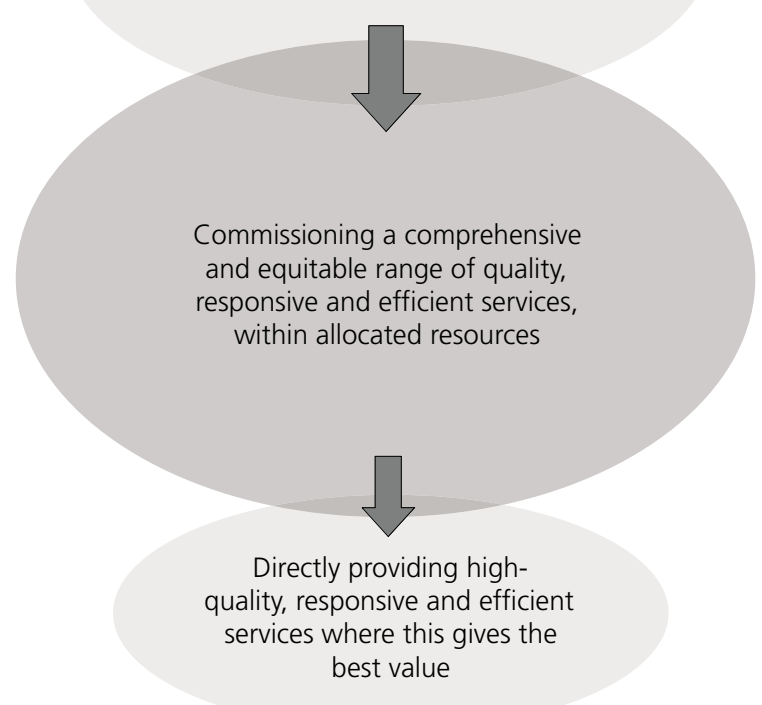

The similarities and differences among these definitions underscore the variety of perspectives that characterize the debate about the role and purpose of commissioning. In particular, they question what emphasis ought to be put on each of the elements of successful commissioning. Wade et al. (2006) underscore the visionary and transactional components, while Øvretveit (1995) stresses the healthgain outcomes. Meantime, the Department of Health model adds the concept of value for money, an essential element in a publicly funded system. The criteria for judging what successful commissioning would add to the health of a local population are, however, less clear. This lack of clarity might reflect the continually changing expectations of healthcare by the public, staff and politicians.

\section{Practice-Based Commissioning}

The final act of devolved decision-making within the Organising Framework for NHS Reforms in England was to devolve purchasing to the lowest organizational level - individual general practitioner (GP) practices. $\mathrm{PBC}$ involves passing funds from a PCT to individual GP practices so that they can commission services for their populations as they see fit and within their PCTs' overall strategic aims (Department of Health 2004). ${ }^{1}$ $\mathrm{PBC}$ is aimed at enhancing the prospects of the following (Crisp 2005):

- The design of improved patient pathways

- A better working partnership with PCTs to create convenient community-based services

- GPs taking responsibility for the budgets delegated by their PCTs, which cover acute, community and emergency care

- More effective budget management

Studies examining the efficacy of effective commissioning by PCTs indicate that the 
following factors are important (Smith et al. 2005):

- Stability in health organizations

- Time for clinical engagement

- Policies that support patient choice

- Policies to allow shifts of resources both between providers and between different sectors

- Incentives for GPs to develop new forms of care

- Effective management support and information

- Regulations to minimize potential conflicts of interest arising from GPs being both commissioners and providers

While compelling, these factors and the seed of reform and innovation are fraught with the following complexities when considered at the level of implementation:

- How to provide stability for healthcare organizations when the prevailing climate has favoured repetitive reconfiguration

- How to deliver effective management support when cost constraints are a constant feature of public service management (as part of the implementation of Creating a Patient-Led NHS, managementcost reductions of $15 \%$ are required)

- How to encourage clinical engagement when demands on GPs are expanded through increasing patient expectations of service alongside a change in culture to a better work/life balance

Smith et al. (2005) further postulate that the greatest challenge facing commissioning will be to create a set of incentives that will engage GPs and enable the development of new forms of seamless services for people with long-term conditions that have eluded previous forms of primary care - led commissioning.
In addition to these organizational factors, there are also profound changes in the skills and competencies required of commissioning leaders and managers, whether clinical or general management. One of the most thoughtful exponents of commissioning skill sets, Simon Stevens, president of United Health in the UK and previously health advisor to the prime minister, has remarked on the need for commissioners to have access to a number of tools, such as the following:

- Actuarial design of risk pools and incentives

- Utilization and equity auditing

- Elective care demand management

- Emergency care subsystems redesign (including out-of-hours and community hospital usage)

- Primary care performance profiles

- Medicines management to ensure appropriate prescribing

- Skill-mix redesign, especially at the primary/secondary, health/social care boundary

- Patient and public engagement, including self-care

- Strategies for changing clinical practice, including clinical decision-support methodologies

\section{The Commissioning Cycle}

The Department of Health (2006a) has identified for local commissioners the cycle and descriptors shown in Figure 4 as additional aids for thinking about translating plans into action.

The following sub-sections explain each of the elements presented in Figure 4 (Department of Health 2006a).

\section{Assessing Needs}

Increasingly, assessing needs will be based on more rigorous analytical approaches involving 
Figure 4. The commissioning cycle for health services

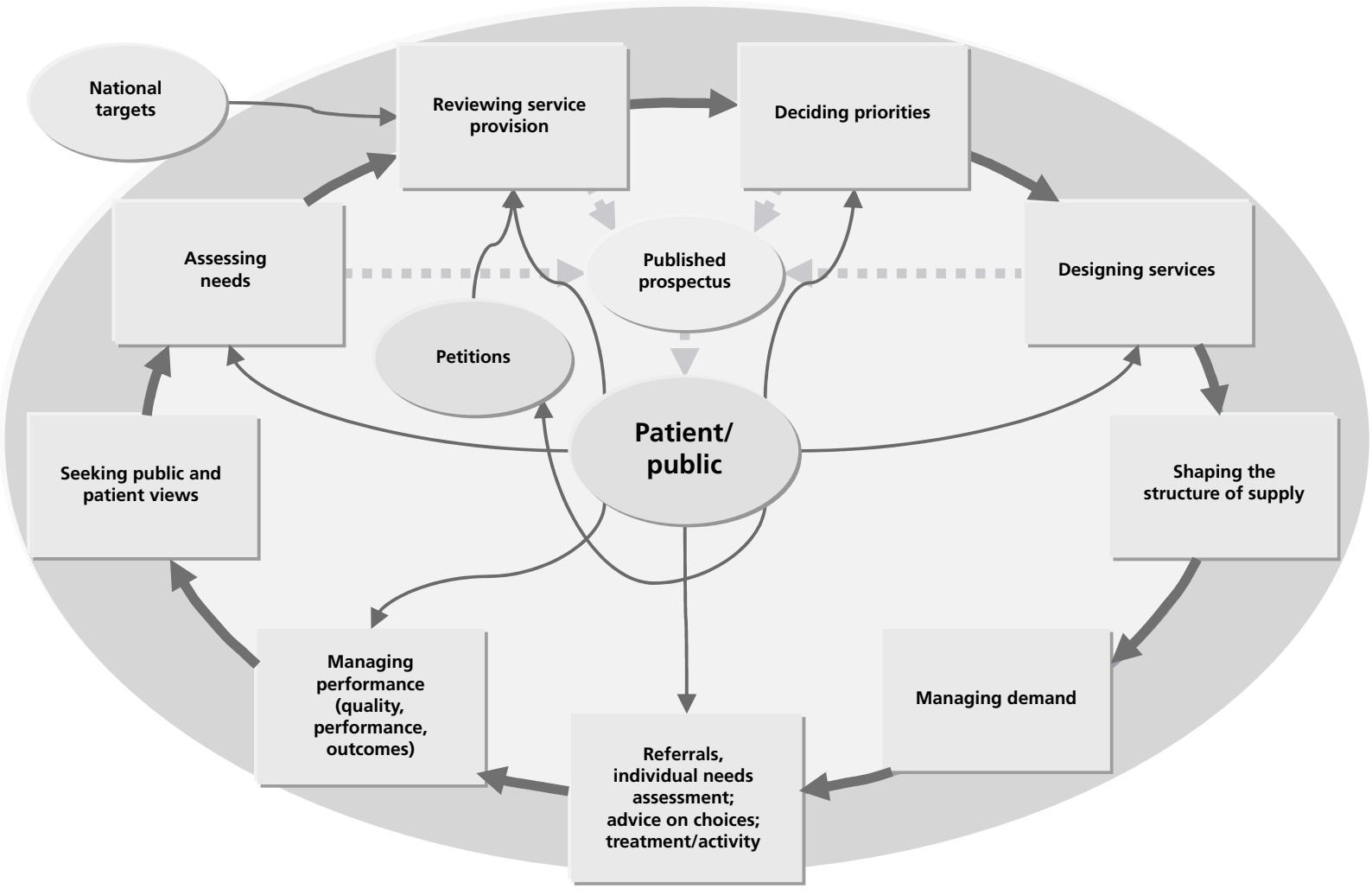

population segmentation and risk stratification. It will involve public health professionals, local authorities, GPs, patients and the local community.

\section{Reviewing Service Provision}

Practices should identify gaps and the potential for improvements in existing services. PCTs should utilize the aggregated intelligence of their practices and their local needs assessment to identify gaps or inadequacies in provision as well as broader requirements for service development.

\section{Deciding Priorities}

PCTs should produce a strategic plan for the health community based on data on needs assessment collated from practices and on the clear choices patients are making. Practices and PCTs are encouraged to work collectively to reinvest resources that have been released through service redesign where these would achieve greater impact. PCTs should ensure their patients and local communities, as well as municipal authorities and other partners, are properly involved in the process of deciding priorities.

\section{Published Prospectus}

Published PCT prospecti will signal the strategic directions for local services, highlighting commissioning priorities, needs and opportunities to service providers. The prospecti offer a focus for discussion with patients and local communities and an opportunity to open dialogues with potential providers.

\section{Designing Services}

Practices are expected to work individually, or in groups, to develop strategies and service 
models to improve healthcare services for the public and to address its priorities.

\section{Shaping the Structure of Supply}

PCTs should be clear about the services and service specifications they and their practices and patients want to see developed and give strategic support to proposals where necessary. PCTs have a role in encouraging and supporting practices that offer services locally and attract private sector and third sector (voluntary) providers to offer services in line with identified needs and priorities. Incentives and levers should be used by PCTs to stimulate the supply of services. PCTs will make contracts with local secondary care providers within a new national contracting framework, with the involvement of practice-based commissioners. For a few very specialized services, contracts will be held at the national level. For other specialized services, PCTs should group together to set contracts.

\section{Managing Demand}

Practices and PCTs will establish strategies for care and resource utilization to ensure that patients receive the most appropriate care in the right setting. This will guarantee that the benefits of healthcare resources are maximized.

\section{Referrals; Individual Needs Assessment; Advice on Choices; Treatment/Activity} Individual practices and clinicians undertake individual needs assessments, make referrals and advise patients on choices and the treatments available to them - each referral is effectively a micro-commissioning decision. Practices must work with social service and other agencies to facilitate the opportunity for patients to make their choices with the benefit of good advice from their GPs.

\section{Managing Performance}

Practices will always seek to manage their budgets to maximize the benefits of the resources available to them. To help them, PCTs have a responsibility to provide support programs, including training and development, developing systems to allow practices to monitor the services their patients receive through accurate, relevant and timely data. PCTs remain responsible for the aggregated financial position and for ensuring overall financial balance.

\section{Seeking Public and Patient Views}

PCTs will be responsible for measuring and reporting on patients' experiences. Practices will also want to monitor patients' satisfaction. Robust mechanisms for collecting and understanding patients' views will need to be developed by PCTs and made available to practices. Throughout, PCTs must ensure that the public voice is heard in the development of priorities and the shaping of services.

\section{Possible Implications of the Organising Framework for NHS Reforms}

No reform program ever has all the facets of its consequences worked out in advance. The Organising Framework raises many questions about the inter-relationship of its components. Work undertaken at the Kings Fund in London, for example, explored a number of key questions about commissioning's ability to effect a shift from secondary to primary care (Palmer 2006):

\section{How will it help cope with potentially unlimited patient demand?}

Demand is unlimited because healthcare is free at the point of need. As the public becomes increasingly health-savvy and assertive, there is a greater likelihood that demands will increase. Palmer (2006) argues that 
commissioning can partly mediate between potential demand and affordability, seeking to obtain the greatest patient benefit with the funds available. Historically, lengthening wait lists/times constituted the pressure valve; however, with the policy drive to reduce wait times this option is no longer available.

In particular, how will PCTs reduce patient demand well below the growth rate seen in recent years - and below the levels required to achieve the access targets - when they have weak levers to manage hospital referrals? The framework says that PCTs and providers should agree on activity levels;

\section{As the public becomes increasingly health-savvy and assertive, there is a greater likelihood that demands will increase.}

however, what happens if they cannot agree or if they do reach agreement but the actual referrals exceed agreed levels? NHS trusts and foundation trusts argue that there is a quasi-market and that they should be free to use available capacity to provide more patient services if patients choose them. They also say they are obliged to make progress toward achieving the access targets. This progress might, however, involve an increase in elective activity that is unaffordable for PCTs and, therefore, inconsistent with PCTs' statutory duty to break even.

\section{How will affordability influence demand?}

If PCTs fail to limit demand they will run up deficits. The key question thus becomes what levers and instruments do they have available to control the volume of hospital referrals? In addition to the above considerations, PCTs will require strong leadership to hold their local markets to account because unpalatable decisions might, in some cases, have to be made about removing certain providers or, at least, exposing them to external competition. The degree to which the government would be willing to see such levels of contestability is as yet unclear. SHAs also have a role in moderating between commissioners and providers over these precise points (Smith et al. 2006). This is also particularly important where "stranded capacity” exists in a system. Palmer (2006) argues that the use of the Private Finance Initiative (PFI) to expand secondary provision will lead to a reduction in the need for more new PFI schemes to be approved.

Where stranded capacity exists, hospitals with annual financial balance targets tend to close this down to save costs. In an unfettered market, the price would fall and the utilization rate would rise as demand rose. If this were allowed in the NHS, Palmer (2006) suggests that commissioners would be able to purchase more services for patients, and providers would be able to provide more services, thereby earning more income and reducing deficits. The result would be less stranded capacity and fewer services closed. Arguably, the NHS can work effectively only when downward price flexibility is allowed (Kings Fund 2006).

\section{What are the incentives for hospitals to increase admissions?}

Hospital providers receive payment for almost all elective procedures under the fixed tariff within the payment-by-results $(\mathrm{PbR})$ system. It follows, therefore, that in a market system hospitals will seek to expand their revenue base to allow for investment in new services by increasing the numbers of "profitable" patients they can see and treat under $\mathrm{PbR}$. However, a number of key constraints apply: 
- Because they are required to consult with their PCT before taking any action, it is difficult for a provider simply to drop "unprofitable" services. Each PCT, not the providers, has the statutory right to consult on service alterations, especially when a change is seen as substantial. The PCT, not the provider, consults with the Local Municipal Authority Health Overview and Scrutiny Committee (OSC) on the proposal. If the OSC objects, it can refer the proposed change to central government.

- The provider may face relatively long leadin times to bring on extra capacity, both physical (e.g., beds, operating theatres) and human resource. This step change in capacity is high risk unless it can be shed according to changing circumstances.

- PBC encourages GPs to hold funds for close-to-home care and encourages the development of joint protocols with secondary providers to ensure that only those patients who need acute care are referred. Providers are more likely to respond well to sophisticated commissioning if they are confident in the future of strategic planning - even if it does not maximize their income.

- The development of a national framework of clinical governance places considerable emphasis on clinical quality and standards. Because providers are subject to independent assessment by the Commission for Healthcare Audit and Inspection, a going-for-everything-you-can-get strategy carries additional risks.

It is important to understand that neither additional nor less work comes at marginal cost. The extra revenue a hospital gains for doing more work - or loses for doing less work - is still paid at the average cost for treating patients. While there will undoubt- edly be marginal costs of treating one patient, work on that case will still be paid at the average cost. Expansion and contraction therefore carry the same management issues of complexity. Equally, there might come a point at which a hospital faces expensive step-costs of bringing on new facilities or resources to cope with extra demand. However, each extra case will still be funded at the average cost. Work by Palmer (2006), based on unpublished data, indicates that the typical hospital cost structure is about $10 \%-15 \%$ variable costs, about $60 \%-75 \%$ semi-fixed costs and about $15 \%-25 \%$ fixed costs.

\section{How are PCTs to shift funding from exist- ing hospital providers to support the provi- sion of new services closer to home?}

The Organising Framework recognizes the need to fund providers of new services closer to home and, in some cases, to offer them incentives. It does not, however, address the fact that PCTs can do this only if they are sure that spending on hospital care will be reduced. Because PCTs have no effective levers to bring about a reduction of revenue spending on hospital care, they are not in a position to contract with new providers to purchase new services. That is because the new services will be affordable for PCTs only if hospital spending goes down, thereby releasing funds to pay for them. This problem is particularly acute for PCTs that are in deficit and/or under strong financial pressure. PCTs cannot expect any help from hospital trusts to reduce hospital demand because that would make it even more difficult for them to achieve financial balance. This situation results in a vicious circle because if there were alternative cheaper services closer to home to which GPs could refer it would be much easier for GP practices and PCTs to manage hospital referrals. These systems are not yet mature; therefore, elective demand remains difficult to manage. 
Consequently, a high proportion of PCTs' budgets is spent on hospital care, making it harder for them to afford to commission or provide alternative services closer to home. PBC can - and should - motivate greater and more rapid development in this direction.

\section{How are PCTs to deliver the commission- ing priorities they identify in their strategic plans for the medium term?}

The description of the commissioning cycle in the framework clearly envisages a strategic commissioning role for PCTs, one that involves deciding priorities in collaboration with local stakeholders and then shaping the structure of supply to ensure that the pattern of expenditure reflects those priorities. A PCT might want to spend more on disease prevention, cancer services or care closer to home; however, if patients exercise choice for more elective hospital care and all the PCT's budget is used to fund this care at full tariff, then there is little the PCT can do about it. This sort of weak commissioning regime leaves the level and pattern of service provision largely to the market. Demand is determined by short-term GP referral practices and supply is determined by the response of existing providers (who are aiming to maximize admissions) to the incentives embedded in the tariffs. Over time, the demand for elective services to hit wait time targets and for technologies is always likely to outstrip available funding - particularly from 2008 when the Wanless effect begins to be felt and funding growth slows sharply.

\section{Some Possible Solutions}

There are many possible refinements that can minimize such consequences; e.g., moving from annual to longer-term contracts and paying the full tariff only for contracted volumes of activity and at a standard marginal cost thereafter. Such an approach could have many advantages:

- Commissioners would be seen to have real teeth through their ability to shift resources.

- Providers would be more likely to support the care-closer-to-home agenda as their ability to expand beyond contracted volumes became more muted. They would also be under a statutory obligation to work in partnership with their PCTs and Health Overview and Scrutiny committees.

- Medium-term contracts would provide greater certainty for providers. This might be less destabilizing for both providers and commissioners because marginal costs would be less severe.

- The incentives for providers to improve efficiency would be greater because of marginal income derived only from extra work.

Following Palmer (2006), however, I should note that some observers might feel that in a market-based system such matters should be left entirely to the market. These same observers might also argue that lower marginal prices for above-contract volumes would reduce the incentive to expand supply, which is an important characteristic of a system that has lower wait times and relatively robust room for patient choices.

In addition, the whole basis of the tariff requires reworking. Such reworking involves, for example, the treatment of excluded costs, the treatment of "sunk costs," the methodology for allocating costs across HRGs, the finished consultant episode (FCE)/spell conversion and the impact of any market factors to recognize unusual costs (e.g., land values in London). 


\section{Understanding the Information Needs of PCT Boards}

The issues discussed above illustrate that commissioning - at whatever level - demands strong information in order continually to assess progress. This information can be in many forms and used in many ways, ranging from public health improvement to contract monitoring of providers. This diversity of information and its application suggests the importance of some underpinning principles (Dr Foster 2006):

- All information should

- be clearly and simply presented;

- be forward-looking and present trends;

- be updated in a timely manner according to its purpose and potential volatility;

- direct the boards' attention to significant risks, issues and expectations; and

- provide the level of detail that is appropriate to the boards' roles.

- Strategic information should

- show trends in health needs, provision and patient satisfaction;

- provide forecasts and anticipate future performance issues; and

- encourage an external focus and understanding of the context for reform and local action.

- Information for performance monitoring should

- provide an accurate and balanced picture of current and recent performance, including financial, clinical, regulatory and patient expectations;

- focus on the most important measures of performance and highlight exceptions;

- be appropriately standardized in order to take account of known factors that affect outcomes, such as the age and deprivation of patients; and

- enable comparisons with the performance of similar organizations and health economies.

- The key tests of the success of any information resource for boards will be the extent to which it

- prompts relevant and constructive challenges;

- $\quad$ supports informed decision-making;

- provides early warning of potential financial or other problems; and

- develops all directors' understanding of the organization, the local health economy and its performance.

The method whereby commissioners acquire this information - given that this responsibility rests with PCTs and is not to be delegated to practice-based commissioners - can be contracted out to third-party agencies, a number of which already exist or might enter the market. Wade et al. (2006: 10) highlight this point using the example of the Greater Manchester Commissioning Business Services:

The Commissioning Business Service (CBS) is a new venture developed by the 14 Greater Manchester primary care trusts (PCTs). The CBS has as its mission the sourcing, procurement and contractmanagement of the delivery of quality and cost-effective services. It will provide a service to PCTs, practice based commissioners, local authorities, collaborative commissioners, and even providers who sub-contract services. CBS services will include: the provision of off the shelf or bespoke service specifications; a detailed directory of providers and services; public health and comparative clinical data and intelligence; and the full range of opera- 
tional contract management including contract compliance and resource utilisation analysis. The CBS is planned to become operational in April 2006 and is currently working on business modeling, the development of memoranda of information for each of its business areas, due diligence, the seeking of expressions of interest in providing CBS services, and the detailed design of the organisational model.

\section{Leadership in Commissioning}

The agenda mapped above highlights the significant leadership task for commissioners. The complexity of the task, taken together with the prospect of national elections in the near future and the financial position required by Wanless (2002), creates a hothouse climate for accelerating progress. The successful management of change and of harnessing the various interests, both organizational and professional, will be critical. Such leader attributes will require development throughout the organizations, not just at the most senior levels (Wade et al. 2006).

This organization-deep leadership function mimics the move away from the heroic leader model, so prevalent during recent decades, to a more empowering and engaging style of leader behaviour and change leadership (although one that is no less transforming in its ideology) (Smith 2002; Higgs and Rowland 2005). Additional leader behaviours particularly critical in this situation are as follows (Alimo-Metcalfe and Alban-Metcalfe 2003):

- Showing genuine concern

- Enabling

- Being accessible

- Encouraging change

- Being honest and consistent and acting with integrity
- Being decisive in resolving complex problems

- Leading the organization and inspiring others

- Focusing effort and supporting a developmental culture

- Facilitating change sensitively

The development of clinical leadership and engagement in the new commissioning models at the PCT and PBC levels will be critical to their success. Clinical leaders add knowledge, experience and skills to the commissioning map. Their leadership development must not be left to chance but supported with personal coaching and organizational development nested in a framework of patient and public engagement in the commissioning process and anticipated outcomes.

\section{What if the Model as Conceived Doesn't Deliver?}

Speculating about "what if" might seem a little advanced when the reform program is still so young. However, a number of issues remain on which further work will be needed.

To begin with, "choice" implies that there needs to be headroom in system capacity in order to offer real choice to patients. PCTs will have the task of determining the desirable characteristics of their local health markets, one aspect of which will be the extent of "free capacity" and how that capacity can be accessed equitably. Easing market entry will require using new flexibilities to help encourage new entrants where normal market signals might be insufficient (Department of Health 2006a). These flexibilities include payments above tariff, guaranteed income for providers and reduced capital investment for providers through PCT capital grants or joint ventures.

It will also be important to find ways to deal with market exit. Many hospitals are 
constructed financially and physically on the basis of an historical legacy. They might argue that the volumes of activity are not readily influenced, prices are fixed and wages are set nationally. Hospitals might also contend that they carry significant fixed costs and they must break even each year. Rules for foundation trusts offer greater budgetary flexibility due to their financial framework; however, they still form the minority of hospitals in England. Where a hospital is failing, Palmer (2006) suggests three options:

- Restructure the trust on a stand-alone basis. This might involve the closure of certain services for which sufficient demand no longer exists, merging clinical services across trusts under common management where this is cost effective and expanding out-patient services to address the closer-to-home agenda.

- Close the trust only when it can be shown that it can be achieved consistent with protecting patients, preserving the concept of patient choice and delivering essential services locally, albeit through a possible alternative supplier.

- Transfer the trust to another party, such as another NHS trust or foundation trust, or to an independent provider. In 2007, a foundation trust absorbed a failing NHS trust (Timmins 2007).

Equally, commissioners would need to lead or endorse such activities as those highlighted earlier. This would place PCTs' decision-making very much in the public gaze. PCTs remain largely answerable to the public by proxy rather than directly (although this might change). Work commissioned by the Health Policy Forum has touched on alternative, and perhaps more radical, options, should these be needed (Smith et al. 2006). These include, for example, holding PCTs more accountable to their local populations through quasi-democratic processes by direct elections to PCT boards, or through a stronger mandate on PCTs from municipal authorities. In addition, while providers compete under patient choice, commissioners have locked-in populations - competition among commissioners might therefore be required. It is, however, too early to be judgemental about the rate, pace and success of the current reform program in England.

\section{Conclusion}

The new commissioning within the English NHS is still very much in its early days. There are hopes and dangers in equal measure. The divergence of health policy approaches within the UK's four countries will allow important comparisons to be made among them. In addition, the complexity of the reform agenda, the newness of the commissioning organizations and the need to accelerate organizational and personal development to deliver the health gains the public and politicians expect are not risk free. These health gains will require influential and visionary leadership. Likewise, significant organizational development will be needed to secure the potential in the system in which clinical and public engagement is an integral part of that leadership drive.

If it is successful, England's health system reform agenda will deliver benefits to individuals and communities. Looking further into the future, more radical changes might then be required to optimize those benefits.

\section{Endnote}

1 The English NHS is unusual in its continuing faith in primary care-based organizations to carry out effective purchasing of healthcare services (Smith et al. 2005).

\section{References}

Alimo-Metcalfe, B. and R.J. Alban-Metcalfe. 2003. "Under the Influence." People Management 9: 32-35. 
Alvarez-Rosete, A., G. Bevan, N. Mays and J. Dixon. 2005. "Effect of Diverging Policy across the NHS." BMJ 331: 946-50.

Crisp, N. 2005. Commissioning a Patient-Led NHS. London: Department of Health.

Department of Health. 2000. The NHS Plan: A Plan for Investment, a Plan for Reform. London: Author. Retrieved May 31, 2007. <http://www.dh.gov.uk/en/ Publicationsandstatistics/Publications/Publications PolicyAndGuidance/DH_4002960>

Department of Health. 2004. NHS Improvement Plan: Putting People at the Heart of Public Services. London: The Stationery Office. Retrieved May 31, 2007. <http://www.dh.gov.uk/en/Publicationsandstatistics/ Publications/PublicationsPolicyAndGuidance/DH_ 4084476>

Department of Health. 2005. Creating a Patient-Led NHS: Delivering the NHS Improvement Plan. London: Author. Retrieved May 31, 2007. <http://www.dh.gov. $\mathrm{uk} / \mathrm{en} /$ Publicationsandstatistics/Publications/ PublicationsPolicyAndGuidance/DH_4106506>

Department of Health. 2006a. Health Reform in England: Update and Commissioning Framework. London: Author. Retrieved May 31, 2007. <http:// www.dh.gov.uk/en/Publicationsandstatistics/ Publications/PublicationsPolicyAndGuidance/DH_ 4137229>

Department of Health. 2006b. PCT and SHA Roles and Functions. London: Author. Retrieved May 31, 2007. <http://www.dh.gov.uk/en/Publicationsand statistics/Publications/PublicationsPolicyAnd Guidance/DH_4134649>

Dr Foster. 2006. The Intelligent Commissioning Board. London: Author.

Greer, S. 2004. Territorial Politics and Health Policy. Manchester: Manchester University Press.

Healthcare Financial Management Association. 2006. Introduction to Health Service Finance for NonExecutive Directors. Bristol: Author.

Higgs, M. and D. Rowland. 2005. "All Changes Great and Small: Exploring Approaches to Change and Its Leadership." Journal of Change Management 5(2): $121-51$.

Kelman, S. 2005. Unleashing Change: A Study of Organizational Renewal in Government. Washington, DC: Brookings Institution Press.

Kings Fund. 2006. Designing the "New" NHS. London: Author.
Klein, R. 2001. The New Politics of the NHS. Harlow: Prentice Hall.

Lewis, R. and J. Dixon. 2005. NHS Market Futures: Exploring the Impact of Health Service Market Returns. London: Kings Fund.

Osborn, R.N. and T. Gaebler. 1992. Reinventing Government: How the Entrepreneurial Spirit is

Transforming the Public Sector. Reading, MA: Addison Wesley.

Øvretveit, J. 1995. Purchasing for Health: A Multidisciplinary Introduction to the Theory and Practice of Purchasing. Buckingham: Open University Press.

Palmer, K. 2006. NHS Reform: Getting Back on Track. London: Kings Fund.

Pollitt, C. 2002. "Clarifying Convergence: Striking Similarities and Durable Differences in Public Management Reform." Public Management Review 4(1): 471-92.

Smith, J., J. Dixon, N. Mays, H. McLeod, N. Goodwin, S. McClelland, R. Lewis and S. Wyke. 2005. "Practice-Based Commissioning: Applying the Research Evidence.” BMJ 331: 1397-99.

Smith, J., R. Lewis and T. Harrison. 2006. Making Commissioning Effective in the Reformed NHS. London: Health Policy Forum.

Smith, R. 2002. "Needed: Transformational Leaders.” BMJ 325: 1351.

Stevens, S. 2004. "Reform Strategies for the English NHS." Health Affairs 23(3): 7-44.

Timmins, N. 2007, April 2. "Trust Takeover of Failed Hospital Shows Way Forward." Financial Times (FT. com). Retrieved May 30, 2007. <http://www.ft.com/ $\mathrm{cms} / \mathrm{s} / 253 \mathrm{bf6d} 4-\mathrm{e} 0 \mathrm{~b} 6-11 \mathrm{db}-8 \mathrm{~b} 48-000 \mathrm{~b} 5 \mathrm{df10621}$. html>

Wade, E., J. Smith, E. Peck and T. Freeman. 2006. Commissioning in the Reformed NHS: Policy into Practice. Birmingham: University of Birmingham/ NHS Alliance.

Wanless, D. 2002. Securing Our Future: Taking a LongTerm View. London: HM Treasury. 